\title{
Quantum Monte Carlo calculation of the energy band and quasiparticle effective mass of the two-dimensional Fermi fluid
}

\author{
N. D. Drummond and R. J. Needs \\ TCM Group, Cavendish Laboratory, University of Cambridge, J. J. Thomson Avenue, Cambridge CB3 OHE, United Kingdom
}

(Received 2 November 2009; published 3 December 2009)

\begin{abstract}
We have used the diffusion quantum Monte Carlo method to calculate the energy band of the twodimensional homogeneous electron gas (HEG), and hence we have obtained the quasiparticle effective mass and the occupied bandwidth. We find that the effective mass in the paramagnetic HEG increases significantly when the density is lowered, whereas it decreases in the fully ferromagnetic HEG. Our calculations therefore support the conclusions of recent experimental studies [Y.-W. Tan et al., Phys. Rev. Lett. 94, 016405 (2005); M. Padmanabhan et al., Phys. Rev. Lett. 101, 026402 (2008); T. Gokmen et al., Phys. Rev. B 79, 195311 (2009)]. We compare our calculated effective masses with other theoretical results and experimental measurements in the literature.
\end{abstract}

DOI: $10.1103 /$ PhysRevB.80.245104

PACS number(s): 71.10.Ay, 71.10.Ca

\section{INTRODUCTION}

Landau's Fermi-liquid theory ${ }^{1}$ is an immensely successful and widely used framework for understanding the properties of interacting electron systems. ${ }^{2}$ Low-energy excitations in a fluid of interacting electrons can be treated as excitations of independent quasiparticles, whose energy-momentum relationship (the quasiparticle energy band) generally differs from that of free electrons. Close to the Fermi surface, the quasiparticle band can be approximated by the free-particle form appropriate for particles of mass $m^{*}$, where the quasiparticle effective mass $m^{*}$ may differ from the bare mass of an electron. Given the widespread use of Fermi-liquid theory, it comes as something of a surprise to learn that the effective mass of a paramagnetic two-dimensional (2D) homogeneous electron gas (HEG) has been the subject of great controversy in recent years. Early experiments ${ }^{3,4}$ found a large enhancement of the effective mass at low density, but subsequent experiments ${ }^{5,6}$ have found the increase in the effective mass to be considerably smaller. On the theoretical side, manybody perturbation theory $(G W)$ calculations give a range of possible results depending on the choice of effective interaction and whether or not the Dyson equation is solved self-consistently, 2,7,8 while quantum Monte Carlo (QMC) studies have found either much less ${ }^{9,10}$ or much greater ${ }^{11}$ enhancement of the effective mass than the experiments suggest. Finally, some recent experiments ${ }^{6}$ have shown that the effective masses in paramagnetic and ferromagnetic HEGs behave quite differently as a function of density, as had been predicted using many-body perturbation theory. ${ }^{12}$ The experiments show that the effective mass of a ferromagnetic HEG decreases as the density is lowered, which has also been observed in a recent $G W$ study. ${ }^{13}$ Understanding the magnetic behavior of the 2D HEG at low density will play an important role in the design of spintronic devices.

In this article, we present QMC calculations ${ }^{14}$ of the energy band of the 2D HEG. We have calculated the band $\mathcal{E}(k)$ by evaluating the difference in the total energy when an electron is added to or removed from a particular momentum state k. To our knowledge, this is the first QMC calculation of the complete 2D HEG occupied energy band. As ex- plained above, electronic excitations close to the Fermi surface correspond to quasiparticle excitations. The electronic and quasiparticle bands therefore agree near the Fermi surface and have the same derivative at $k_{F}$. The effective mass of a HEG can be written $\operatorname{as}^{2} m^{*}=k_{F} /(\partial \mathcal{E} / \partial k)_{k_{F}}$, where $k_{F}$ is the Fermi wave vector, ${ }^{15}$ and hence it is straightforward to compute the effective mass once the energy band has been determined. Our effective mass data should help to resolve the controversies surrounding the increase in the effective mass of the paramagnetic 2D HEG at low density. We have studied both paramagnetic and ferromagnetic HEGs in order to look for the differences in behavior observed by Padmanabhan et al. ${ }^{6}$

We use Hartree atomic units $\left(\hbar=|e|=m_{e}=4 \pi \epsilon_{0}=1\right)$ throughout. Densities are given in terms of the radius $r_{s}$ of the circle that contains one electron on average. All our QMC calculations were performed using the CASINO code. ${ }^{16}$

The rest of this article is arranged as follows. We explain our methodology in Sec. II. We present our results in Sec. III. Finally we draw our conclusions in Sec. IV.

\section{METHODOLOGY}

In the variational quantum Monte Carlo (VMC) method, expectation values are calculated with respect to a trial wave function, the integrals being performed by a Monte Carlo technique. In the diffusion quantum Monte Carlo $^{14}$ (DMC) method the imaginary-time Schrödinger equation is used to evolve an ensemble of electronic configurations towards the ground state. Fermionic symmetry is maintained by the fixed-node approximation, ${ }^{17}$ in which the nodal surface of the wave function is constrained to equal that of a trial wave function.

Our trial wave functions consisted of Slater determinants of plane-wave orbitals multiplied by a Jastrow correlation factor. The Jastrow factor contained polynomial and planewave expansions in electron-electron separation. ${ }^{18}$ The orbitals in the Slater wave function were evaluated at quasiparticle coordinates related to the actual electron positions by backflow functions consisting of polynomial expansions in 
electron-electron separation. ${ }^{19}$ The wave functions were optimized by variance minimization ${ }^{20,21}$ followed by linearleast-squares energy minimization. ${ }^{22}$ The high quality of our trial wave functions is demonstrated in this paper and in Ref. 23.

The single-particle energy for an occupied state at wave vector $\mathbf{k}$ is defined to be $\mathcal{E}(\mathbf{k}) \equiv E_{0}-E_{-}(\mathbf{k})$, while the singleparticle energy for an unoccupied state is $\mathcal{E}(\mathbf{k}) \equiv E_{+}(\mathbf{k})-E_{0}$, where $E_{0}$ is the ground-state total energy, $E_{+}(\mathbf{k})$ is the total energy of the system with an extra electron placed in orbital $\exp (i \mathbf{k} \cdot \mathbf{r}),{ }^{24}$ and $E_{-}(\mathbf{k})$ is the total energy with an electron removed from orbital $\exp (i \mathbf{k} \cdot \mathbf{r})$. In a finite simulation-cell subject to periodic boundary conditions, the available states $\{\mathbf{k}\}$ fall on the grid of reciprocal-lattice points offset by the simulation-cell Bloch vector $\mathbf{k}_{s} \cdot{ }^{25,26}$ The simulation cell was left unchanged when electrons were added or removed. We have confirmed that finite-size biases are negligible by carrying out simulations in different cell sizes: see Figs. 2 and 3. We have also verified that the band values obtained with different simulation-cell Bloch vectors lie on the same curve as the band values obtained with $\mathbf{k}_{s}=\mathbf{0}$. Having determined the energy band at a series of $k$ values, we performed a least-squares fit of a quartic function $\mathcal{E}(k)=\alpha_{0}+\alpha_{2} k^{2}+\alpha_{4} k^{4}$ to the band values. The DMC energy band is defined as a difference in total-energy eigenstates; as explained in the introduction, this coincides with the quasiparticle band near the Fermi surface and hence gives a correct description of the effective mass.

The number of electrons $N$ in each of our ground-state calculations was chosen to be a "magic number" corresponding to a closed-shell configuration when $\mathbf{k}_{s}=\mathbf{0}$. In this case real, single-determinant wave functions are appropriate for the ground-state calculations, facilitating the optimization of the wave function. In the $(N+1)$ - and $(N-1)$-electron excited-state calculations, we used the Jastrow factor and backflow function that were optimized for the $N$-electron ground state. Reoptimizing the wave function in the excited state was not found to make a significant difference to the VMC or DMC energies. To try to obtain a better estimate of the energy of the $(N+1)$-electron system, we constructed a multideterminant wave function in which the extra electron occupied each of the symmetry-equivalent $\mathbf{k}$ vectors in the partially filled shell. The determinant coefficients were free parameters, which we optimized by linear-least-squares energy minimization. However, we were unable to lower the VMC energy significantly using this form of wave function, so in our production calculations we used single-determinant wave functions for the $(N+1)$ - and $(N-1)$-electron systems. Our DMC results are converged with respect to time step, as is clear from the agreement between the energy bands obtained at different time steps in Fig. 2(b).

Unlike the DMC calculations of Kwon et al.,,${ }^{9,10}$ we did not promote electrons from the ground-state configuration; we simply added or subtracted single electrons. The energy difference that results from promoting an electron contains a contribution from the interaction between the excited electron and the hole that it leaves behind, in addition to the difference of band energies. By contrast, the energy difference resulting from adding or subtracting an electron simply gives the corresponding band energy. We believe our ap- proach to be a simpler procedure for calculating the band and hence the effective mass, although it does not give values for the quasiparticle-interaction Fermi-liquid parameters. Our determination of the effective mass will facilitate subsequent calculation of the other Fermi-liquid parameters using the approach of Kwon et al.

The other important difference between our methodology and that of Kwon et al. is that we have used a fit to the entire occupied band to determine the derivative at $k_{F}$ and hence the effective mass. This was done for the following reasons: (i) evaluating the derivative numerically using only a few band values near $k_{F}$ is unreliable because of the noise in the band data; (ii) the DMC-calculated band suffers from Hartree-Fock-like pathological behavior in the vicinity of $k_{F}$ because the method does not retrieve all the correlation energy (see the discussion in Sec. III B); and (iii) the band evaluated in a finite cell may suffer from finite-size effects in the vicinity of the Fermi surface. ${ }^{11}$ Although the pathological behavior dominates the derivative of the band in the vicinity of $k_{F}$, it has only a negligible effect on the band fitted over a wide range of $k$. The fit to the band is good, so that the derivative of the fitted band at $k_{F}$ should be reliable: see Figs. 2 and 3 and Figs. 8 and 9.

The occupied bandwidth of the HEG is $\Delta \mathcal{E}=\mathcal{E}\left(k_{F}\right)-\mathcal{E}(0)$ $=E_{-}(0)-E_{-}\left(k_{F}\right)$. The DMC bandwidth is expected to be an upper bound on the true bandwidth: assuming that DMC retrieves the same fraction of the correlation energy in the ground and excited states, the bandwidth will lie between the Hartree-Fock $(\mathrm{HF})$ value $E_{-}^{\mathrm{HF}}(0)-E_{-}^{\mathrm{HF}}\left(k_{F}\right)$, which is too large, ${ }^{2}$ and the exact result $E_{-}^{\text {exact }}(0)-E_{-}^{\text {exact }}\left(k_{F}\right)$. Likewise, the Slater-Jastrow DMC bandwidths are expected to be greater than the Slater-Jastrow-backflow DMC bandwidths, as can be seen to be the case in Fig. 2(b). To obtain an accurate bandwidth, it is essential to retrieve a very large fraction of the correlation energy in the QMC calculations, which explains why the use of DMC and the inclusion of backflow is so important in this work.

A crude way of estimating the ground-state energy is to plot the VMC energy against the variance obtained with different trial wave functions and extrapolate the VMC energy linearly to zero variance, as shown in Fig. 1. This procedure suggests that our DMC calculations retrieve more than $99 \%$ of the correlation energy, and that the fraction retrieved is similar in both the ground-state and excited-state calculations. Suppose the free-electron bandwidth is greater than or approximately equal to the exact bandwidth (this is true for the ferromagnetic HEG and approximately true for the paramagnetic HEG), so that the error in the HF bandwidth is less than or approximately equal to $\Delta \mathcal{E}^{\mathrm{HF}}-\Delta \mathcal{E}^{\text {free }}=k_{F}(1-2 / \pi)$. Hence the error in the DMC bandwidth is less than $0.01 k_{F}(1-2 / \pi) \approx 0.007 / r_{s}$ for a ferromagnetic HEG and less than about $0.01 k_{F}(1-2 / \pi) \approx 0.005 / r_{s}$ for a paramagnetic HEG. Since the bandwidth falls off as $r_{s}^{-2}$, the error is more significant at large $r_{s}$. In the worst case, the paramagnetic HEG at $r_{s}=10$ a.u., this argument suggests that DMC overestimates the bandwidth by about $9 \%$. The errors in the other results are much smaller. It seems reasonable to assume that if the bandwidth is overestimated by a given amount then the effective mass will be underestimated by a similar fraction. 


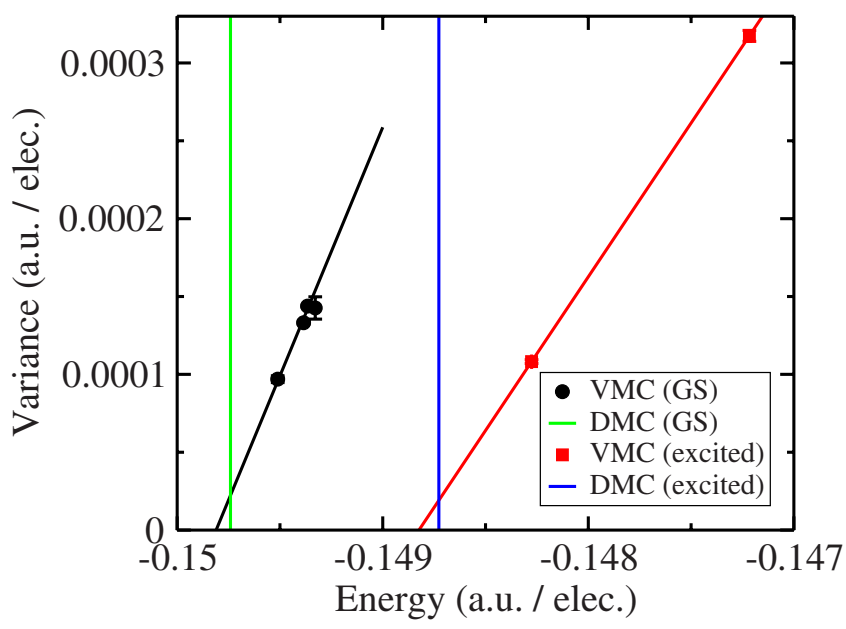

FIG. 1. (Color online) VMC variance against energy for different trial wave functions for a 58-electron paramagnetic HEG of density parameter $r_{s}=5$ a.u. Plots are shown for the GS and an excited state in which an electron is removed from the highestoccupied shell. The slanted lines show fits to the VMC data. The vertical lines show the fixed-node DMC energies obtained with Slater-Jastrow-backflow wave functions. The HF ground-state and excited-state energies are -0.100222 and -0.099632 a.u. per electron, respectively. The difference between the slanted and vertical lines at zero variance gives an approximation to the correlation energy missing in the DMC calculation. This is clearly small compared with the difference between the HF and DMC energies.

\section{RESULTS}

\section{A. Energy bands}

Our calculated energy bands are shown in Figs. 2 and 3 for paramagnetic and ferromagnetic HEGs, respectively. The free-electron and HF bands are shown for comparison. As is well-known, the free-electron band is very much more accurate than the HF band, especially at low densities and especially in the paramagnetic fluid. The HF band is pathological due to the long range of the exchange hole, which results in incomplete screening of the Coulomb interaction. ${ }^{2}$ Our DMC bandwidths are shown in Table I. The bandwidth in paramagnetic HEGs at intermediate and low densities is less than the free-electron bandwidth. On the other hand, in the ferromagnetic HEG the bandwidth is greater than the free-electron bandwidth at all densities studied. In each case the DMC bandwidth is considerably smaller than the HF bandwidth.

It is striking how closely the DMC band agrees with the free-electron band $\mathcal{E}(k)=k^{2} / 2$ for a paramagnetic HEG at $r_{s}$ $=1$ a.u. At $r_{s}=5$ and 10 a.u., the quartic term $\alpha_{4} k^{4}$ in the fitted band is positive for the paramagnetic HEG. For the ferromagnetic HEG the quartic term is negative at all densities. In either case the band is clearly not quadratic. This will result in nonfree-particle-like thermodynamic behavior at high temperatures.

\section{B. Effective masses}

Our DMC effective masses for paramagnetic and ferromagnetic HEGs are plotted in Figs. 4 and 5, respectively, along with various experimental results and previous theoret-

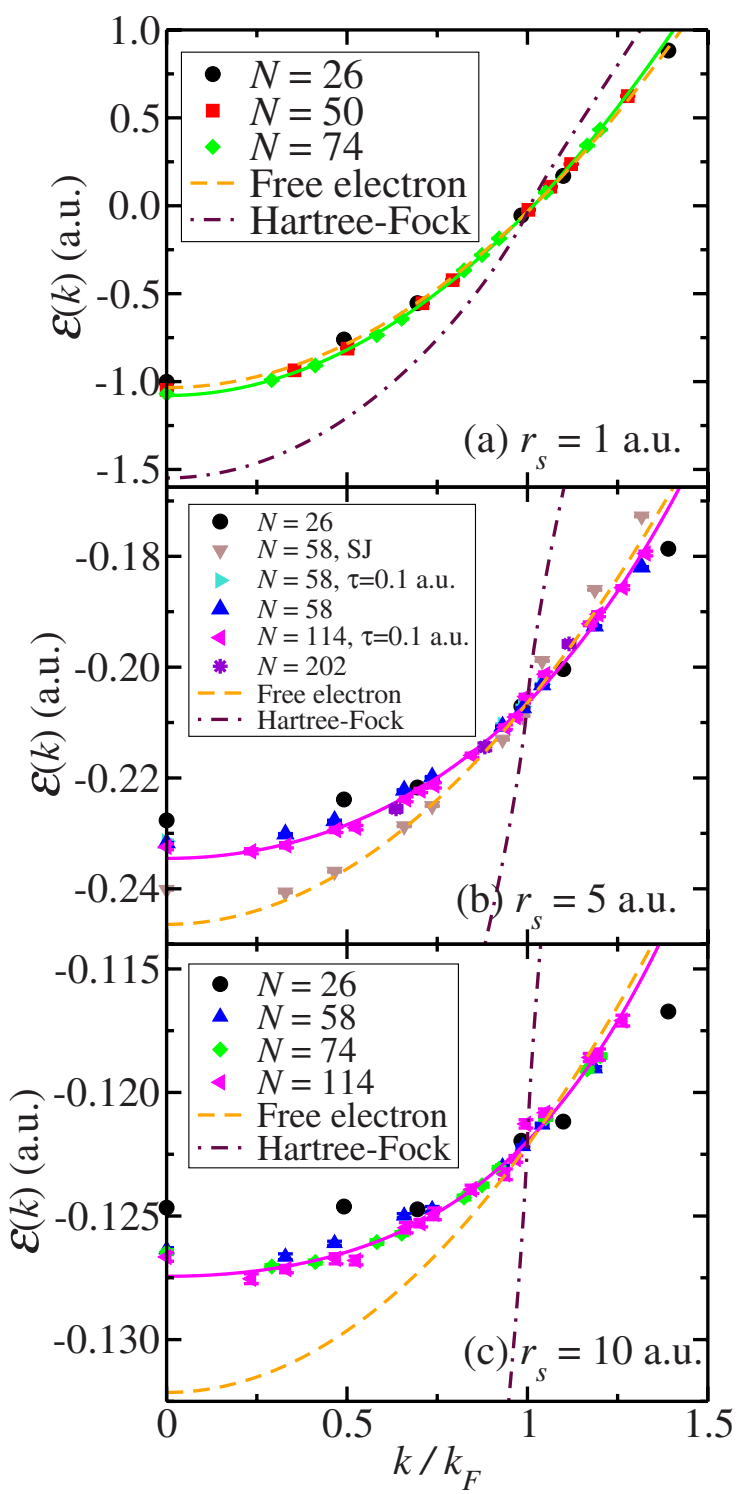

FIG. 2. (Color online) Energy bands of paramagnetic $N$-electron 2D HEGs at $r_{s}=1$ (top), 5 (middle), and 10 a.u. (bottom). The free-electron and HF bands are offset to coincide with the fitted DMC band at $k=k_{F}$. The curve labeled "SJ" used a Slater-Jastrow trial wave function; the others used a Slater-Jastrow-backflow trial wave function. Except where indicated otherwise, DMC time steps $\tau$ of $0.01,0.2$, and 0.4 a.u. were used at $r_{s}=1,5$, and 10 a.u. The solid lines show quartic fits to the DMC data for $N=74,114$, and 114 electrons at $r_{s}=1,5$, and 10 a.u., respectively. (These are the largest system sizes for which we have sufficient data to perform an adequate fit.)

ical predictions. Our effective masses are also given in Table II. In a paramagnetic HEG the effective mass increases with $r_{s}$ : at $r_{s}=1$ a.u. the effective mass is slightly less than the bare electron mass, but at $r_{s}=5$ a.u. the effective mass is significantly enhanced. On the other hand, in ferromagnetic HEGs the effective mass decreases when the density is lowered. Our results therefore support the conclusions of Padmanabhan et al. ${ }^{6}$ In fact our ferromagnetic effective masses are in good quantitative agreement with the experimental data of Padmanabhan et al., ${ }^{27}$ while our paramagnetic effec- 


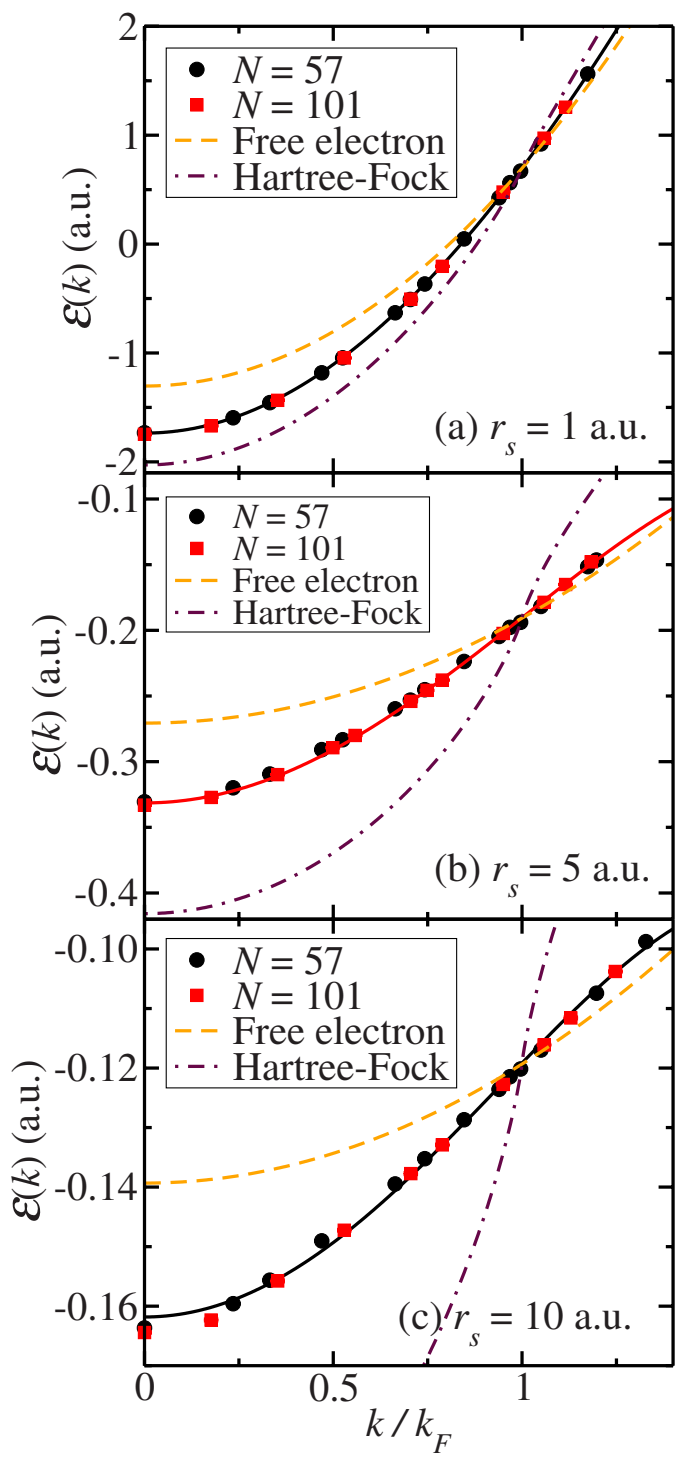

FIG. 3. (Color online) As Fig. 2, but for ferromagnetic HEGs. The solid lines show quartic fits to the DMC data for $N=57,101$, and 57 electrons at $r_{s}=1,5$, and 10 a.u., respectively.

tive masses are in reasonable agreement with those measured by Tan et $a l .{ }^{5}$ We do not find especially good agreement with earlier theoretical work, however. As can be seen in Fig. 4, the effective masses obtained using the $G W$ method depend strongly on the choice of effective interaction and whether or not the Dyson equation is solved self-consistently, undermin-

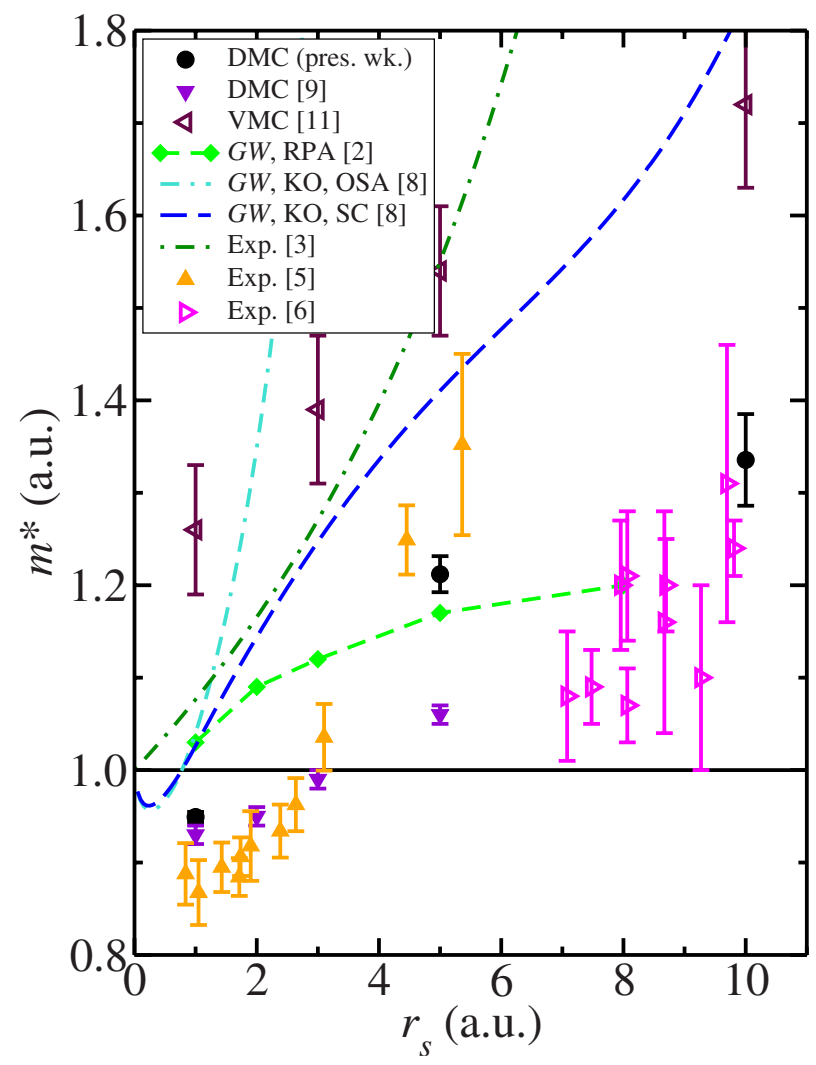

FIG. 4. (Color online) Effective mass $m^{*}$ against density parameter $r_{s}$ for paramagnetic or partially spin-polarized 2D HEGs, as calculated or measured by different authors. Our DMC results were obtained from the fitted curves shown in Fig. 2. The $G W$ results were obtained using the random-phase-approximation effective interaction (Ref. 2) and the Kukkonen-Overhauser (KO) effective interaction (Ref. 8) by solving the Dyson equation self-consistently (SC) or within the on-shell approximation (OSA). All the results shown are for paramagnetic HEGs with the exception of the experimental results of Ref. 6, which are for a partially spin-polarized HEG.

ing confidence in that approach. The DMC data of Kwon et al. do not show a significant enhancement of the paramagnetic effective mass at low densities. ${ }^{9}$ (Our calculations differ from those of Kwon et al. in that we use wave functions that retrieve a greater fraction of the correlation energy, we use larger system sizes, and we use different excitations to evaluate the effective mass.)

TABLE I. Bandwidths of paramagnetic and ferromagnetic 2D HEGs of density parameter $r_{s}$, as calculated using DMC, free-electron theory $\left(\Delta \mathcal{E}=k_{F}^{2} / 2\right)$, and HF theory $\left[\Delta \mathcal{E}=k_{F}^{2} / 2+k_{F}(1-2 / \pi)\right]$. The DMC bandwidths were obtained from the fitted curves shown in Figs. 2 and 3.

\begin{tabular}{|c|c|c|c|c|c|c|}
\hline \multirow{3}{*}{$\begin{array}{c}r_{s} \\
\text { (a.u.) }\end{array}$} & \multicolumn{6}{|c|}{ Bandwidth (a.u.) } \\
\hline & \multicolumn{2}{|c|}{ DMC } & \multicolumn{2}{|c|}{ Free electron } & \multicolumn{2}{|c|}{$\mathrm{HF}$} \\
\hline & Paramagnetic & Ferromagnetic & Paramagnetic & Ferromagnetic & Paramagnetic & Ferromagnetic \\
\hline 1 & $1.045(5)$ & $2.434(6)$ & 1.00 & 2.00 & 1.513 & 2.726 \\
\hline 5 & $0.0281(8)$ & $0.141(1)$ & 0.04 & 0.08 & 0.142 & 0.225 \\
\hline 10 & $0.0055(3)$ & $0.0427(8)$ & 0.01 & 0.02 & 0.061 & 0.092 \\
\hline
\end{tabular}




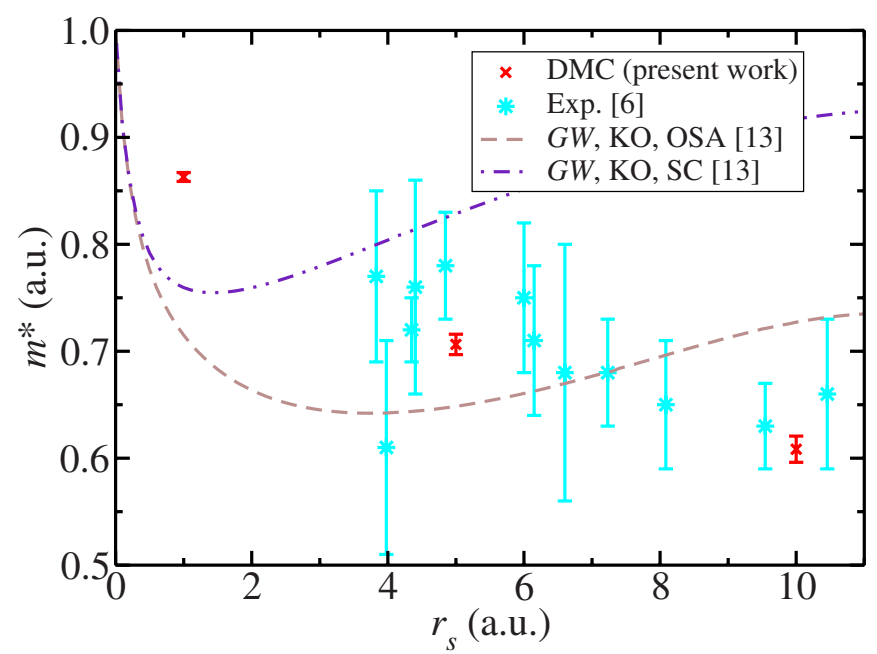

FIG. 5. (Color online) Effective mass $m^{*}$ against density parameter $r_{s}$ for ferromagnetic 2D HEGs. Our DMC results were obtained from the fitted curves shown in Fig. 3. The $G W$ results were obtained using the $\mathrm{KO}$ effective interaction by solving the Dyson equation SC or within the on-shell OSA. (Ref. 13)

Holzmann et al. ${ }^{11}$ have recently studied the paramagnetic 2D HEG effective mass using the VMC method. Their effective masses differ significantly from our DMC results: see Fig. 4. Holzmann et al. considered additions of electrons in the vicinity of the Fermi surface. They found substantial finite-size effects in the effective mass, which they corrected by considering the finite-size dependence of the discontinuity in the momentum distribution at the Fermi edge. Our effective masses do not appear to suffer from these finite-size effects; in fact, our effective masses show the opposite trend with system size, as can be seen in Fig. 6. If the finite-size correction to the mass falls off as $N^{-1 / 4}$ as predicted by Holzmann et al. then the correction should be roughly halved on going from $N=18$ to $N=202$ electrons. Noting that the correction is supposed to increase the mass, this ought to be visible in our data. Since it is not, the finite-size correction of Holzmann et al. appears to be statistically insignificant.

A possible explanation for this difference is that we obtained our effective masses by fitting a band to a wide range of $k$ values instead of just considering the behavior in the vicinity of the Fermi surface. As can be seen in Fig. 7, the HF bands of finite systems as well as infinite ones exhibit pathological behavior near the Fermi surface. The derivatives of the bands become large and fluctuate wildly. The

TABLE II. DMC-calculated effective masses $m^{*}$ of paramagnetic and ferromagnetic 2D HEGs of density parameter $r_{s}$.

\begin{tabular}{ccc}
\hline \hline \multirow{2}{*}{$\begin{array}{c}r_{s} \\
\text { (a.u.) }\end{array}$} & \multicolumn{2}{c}{$m^{*}($ a.u. $)$} \\
\cline { 2 - 3 } 1 & Paramagnetic & Ferromagnetic \\
\hline 5 & $0.949(6)$ & $0.863(4)$ \\
10 & $1.21(2)$ & $0.71(1)$ \\
& $1.34(5)$ & $0.61(1)$ \\
\hline
\end{tabular}

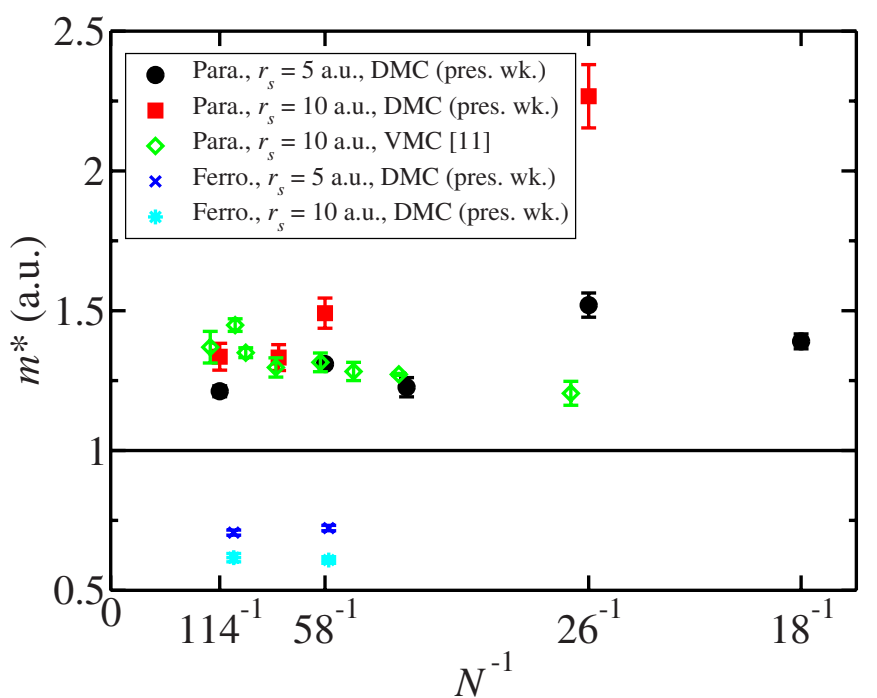

FIG. 6. (Color online) DMC effective mass $m^{*}$ against system size $N$ for paramagnetic 2D HEGs.

numerical derivatives of the DMC bands are plotted in Figs. 8 and 9. It can be seen that the DMC bands exhibit residual Hartree-Fock-like pathological behavior at the Fermi surface, because not all the correlation energy is retrieved. The pathological behavior is more pronounced in paramagnetic HEGs and at low densities. Our procedure of fitting the band over a wide range of $k$ enables us to avoid



FIG. 7. (Color online) HF energy bands (a) and their derivatives (b) for $N$-electron paramagnetic 2D HEGs of density parameter $r_{s}$ $=10$ a.u. 


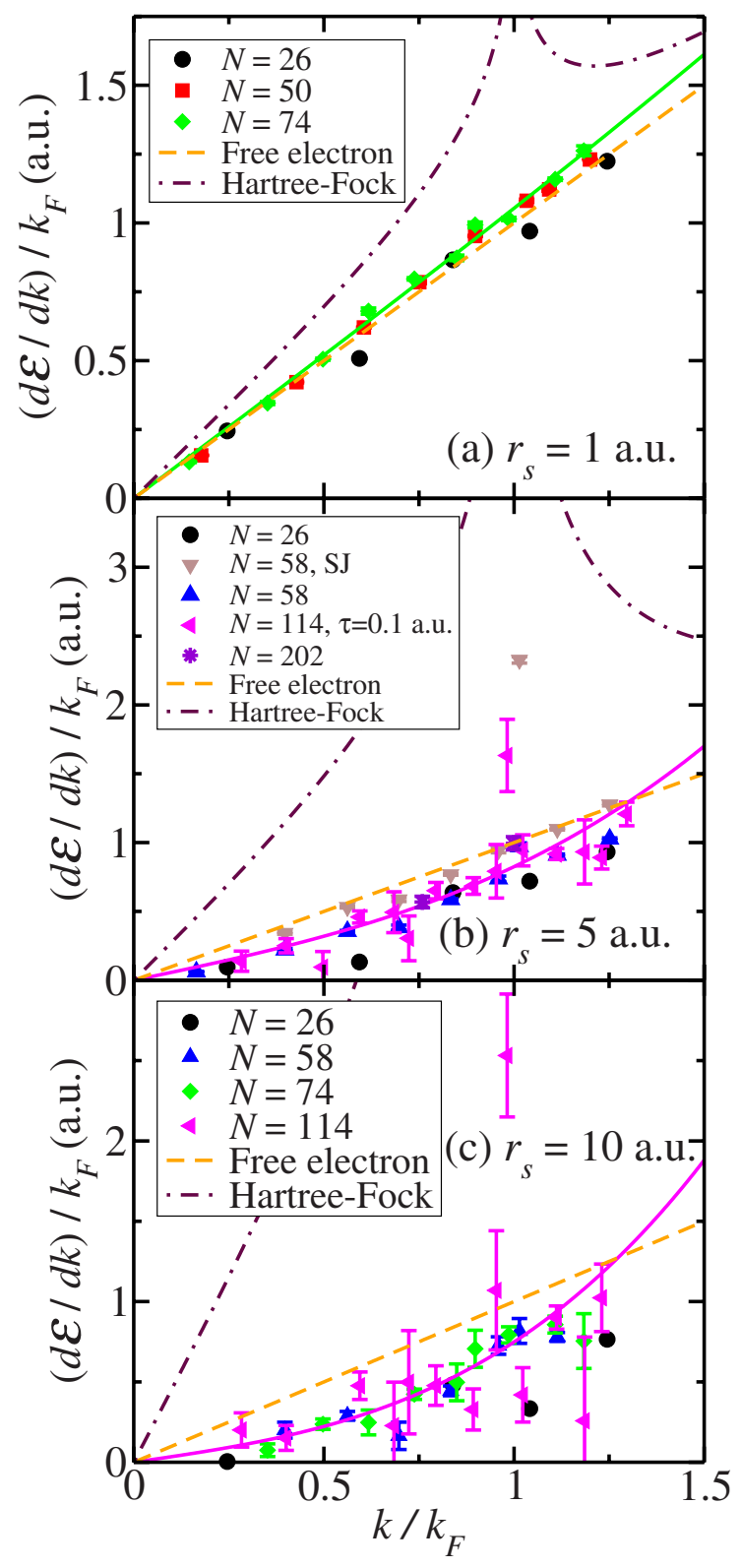

FIG. 8. (Color online) Derivatives of the energy bands of paramagnetic $N$-electron 2D HEGs at $r_{s}=1$ (top), 5 (middle), and 10 a.u. (bottom). The curve labeled SJ used a Slater-Jastrow trial wave function; the others used a Slater-Jastrow-backflow trial wave function. Except where indicated otherwise, DMC time steps $\tau$ of 0.01 , 0.2 , and 0.4 a.u. were used at $r_{s}=1,5$, and 10 a.u. The central difference approximation was used to evaluate the numerical derivatives. The solid lines show the derivatives of quartic fits to the DMC energy bands for $N=74,114$, and 114 electrons at $r_{s}=1,5$, and 10 a.u., respectively.

this pathological behavior. The finite-size effects considered by Holzmann et al. only affect the energy band in the vicinity of the Fermi surface, suggesting that it is also possible to avoid these finite-size effects by fitting the band over a wide range of $k$.

It may seem counterintuitive to use DMC data for excitations far from the Fermi surface in order to determine the effective mass, which is a parameter in a theory describing

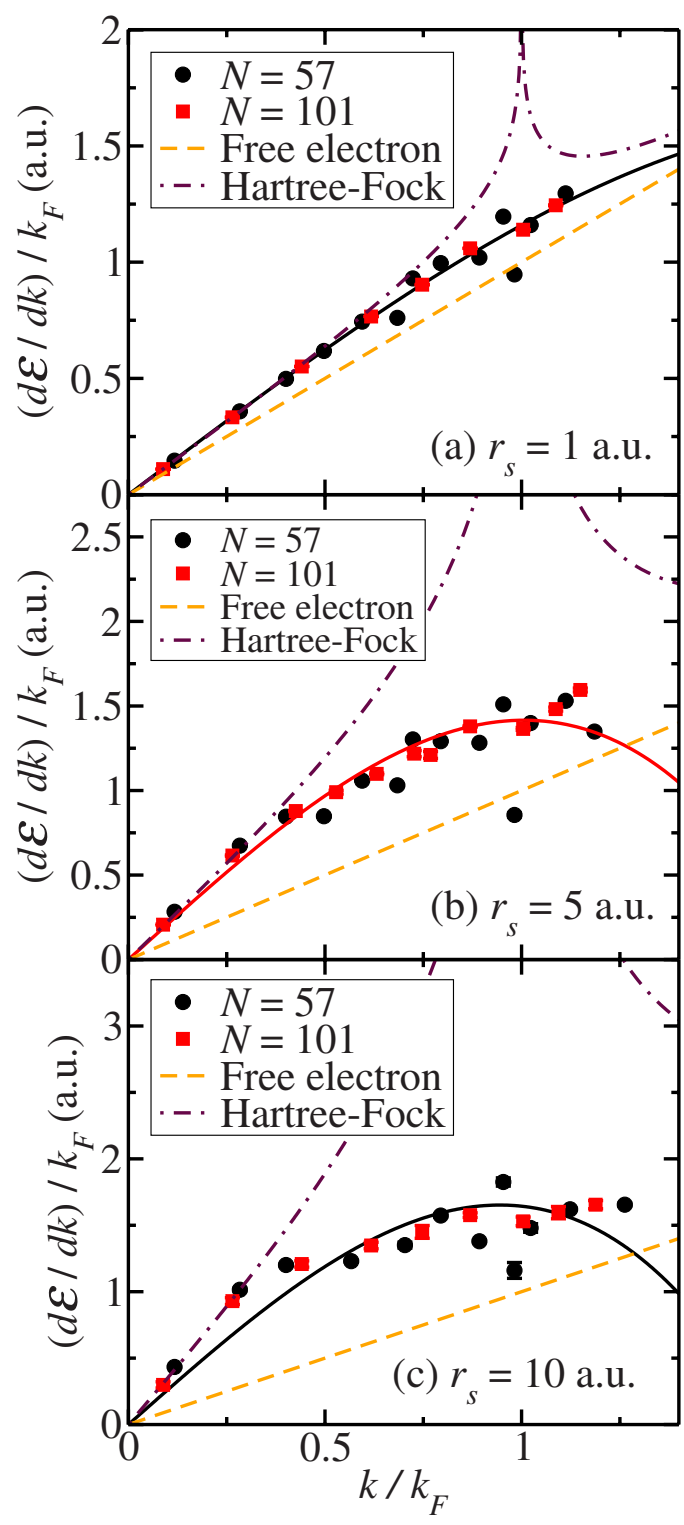

FIG. 9. (Color online) As Fig. 8, but for ferromagnetic HEGs. The solid lines show the derivatives of quartic fits to the DMC energy bands for $N=57,101$, and 57 electrons at $r_{s}=1,5$, and 10 a.u., respectively.

excitations in the vicinity of the Fermi surface. However, we reiterate that it is a premise of Fermi liquid theory that the quasiparticle band coincides with the energy band defined by differences in total-energy eigenvalues in the vicinity of the Fermi surface, so that the derivative of the quasiparticle band at the Fermi surface is equal to the derivative of the electronic energy band. It is possible in principle that the gradient of the energy band may change sharply in the vicinity of the Fermi surface, but there seems to be no reason to suppose this to be the case and our results do not provide any evidence for this sort of behavior: the energy bands shown in Figs. 2 and 3 look well behaved. Nevertheless, it is comforting to observe that our effective mass results are insensitive to a reduction in the range of $k$ values used to perform the fit. This result is obvious from looking at Figs. 2 and 3, and it also follows from a quantitative study, as shown in 
TABLE III. Effective mass $m^{*}$ versus range of $k$ values used to fit the energy band for a 114-electron paramagnetic HEG at $r_{s}$ $=5$ a.u.

\begin{tabular}{ccc}
\hline \hline Range & No. pts in fit & $\begin{array}{c}m^{*} \\
(\text { a.u. })\end{array}$ \\
\hline $0.0 \leq k / k_{F} \leq 1.33$ & 17 & $1.21(2)$ \\
$0.23 \leq k / k_{F} \leq 1.26$ & 15 & $1.18(2)$ \\
$0.33 \leq k / k_{F} \leq 1.20$ & 13 & $1.14(2)$ \\
$0.47 \leq k / k_{F} \leq 1.17$ & 11 & $1.12(3)$ \\
\hline \hline
\end{tabular}

Tables III and IV. For both the paramagnetic and ferromagnetic HEGs, discarding data far from $k_{F}$ has little effect on the calculated effective mass, until the remaining data points are all sufficiently close to $k_{F}$ that either the pathological behavior of the DMC energy band in this region starts to dominate or there are insufficient data to perform an accurate fit.

Another possible reason for not reproducing the finite-size errors in the effective mass predicted by Holzmann et al. might simply be that some of their assumptions are invalid. We have reproduced their $O\left(N^{-1 / 4}\right)$ scaling of the finite-size error in the renormalization factor (the discontinuity $Z$ in the momentum density at the Fermi edge) in VMC calculations, as shown in Fig. 10. However, when an electron is added, there are two contributions to the momentum density: a peak of weight $Z$ at the momentum at which the electron is added and a smeared-out background of weight $1-Z{ }^{2}$ Together, these two contributions to the change in the momentum density are responsible for the change in the kinetic energy when the electron is added, i.e., for the kinetic contribution to the energy band. Examples of the changes in the VMC momentum density that result from adding or removing electrons from different $\mathbf{k}$ are shown in Fig. 11. It can be seen that the smeared-out background depends on the $\mathbf{k}$ at which the electron is added, its average tending to increase with $k$. The finite-size error in the weight $Z$ of the peak is equal and opposite to the finite-size error in the weight $1-Z$ of the background. The finite-size error in the derivative of the energy band due to the background therefore tends to cancel the finite-size error due to the peak. So it is not clear that the $O\left(N^{-1 / 4}\right)$ finite-size error in the renormalization factor should result in an $O\left(N^{-1 / 4}\right)$ error in the gradient of the energy band and hence effective mass.

TABLE IV. Effective mass $m^{*}$ versus range of $k$ values used to fit the energy band for a 101-electron ferromagnetic HEG at $r_{s}$ $=5$ a.u.

\begin{tabular}{ccl}
\hline \hline Range & No. pts in fit & \multicolumn{1}{c}{$\begin{array}{c}m^{*} \\
(\text { a.u. })\end{array}$} \\
\hline $0.0 \leq k / k_{F} \leq 1.18$ & 12 & $0.706(9)$ \\
$0.18 \leq k / k_{F} \leq 1.12$ & 10 & $0.72(1)$ \\
$0.35 \leq k / k_{F} \leq 1.06$ & 8 & $0.722(9)$ \\
$0.5 \leq k / k_{F} \leq 0.95$ & 6 & $0.686(8)$ \\
\hline \hline
\end{tabular}

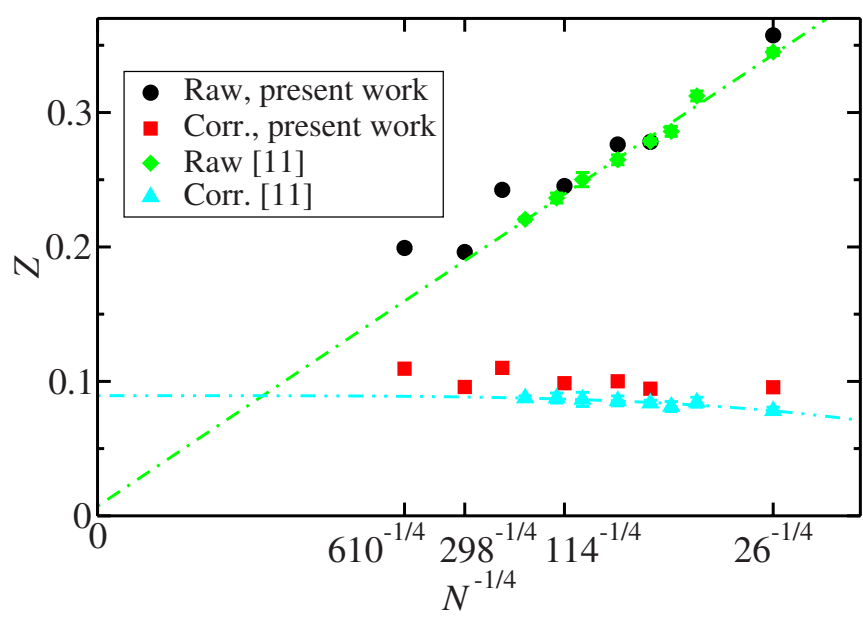

FIG. 10. (Color online) VMC renormalization factor $Z$ against number of electrons $N$ for paramagnetic 2D HEGs at $r_{s}=10$ a.u.

\section{CONCLUSIONS}

In summary, we have used DMC to calculate the energy band of the interacting 2D HEG, and hence we have obtained the quasiparticle effective mass. Our ferromagnetic and paramagnetic effective masses are in agreement with the experimental results of Padmanabhan et al. ${ }^{6}$ and Tan et al.,${ }^{5}$ respectively. In particular, our data confirm that the effective mass of the paramagnetic HEG increases when the density is lowered, while the effective mass of the ferromagnetic HEG decreases.

\section{ACKNOWLEDGMENTS}

We acknowledge financial support from the Leverhulme Trust, Jesus College, Cambridge, and the UK Engineering

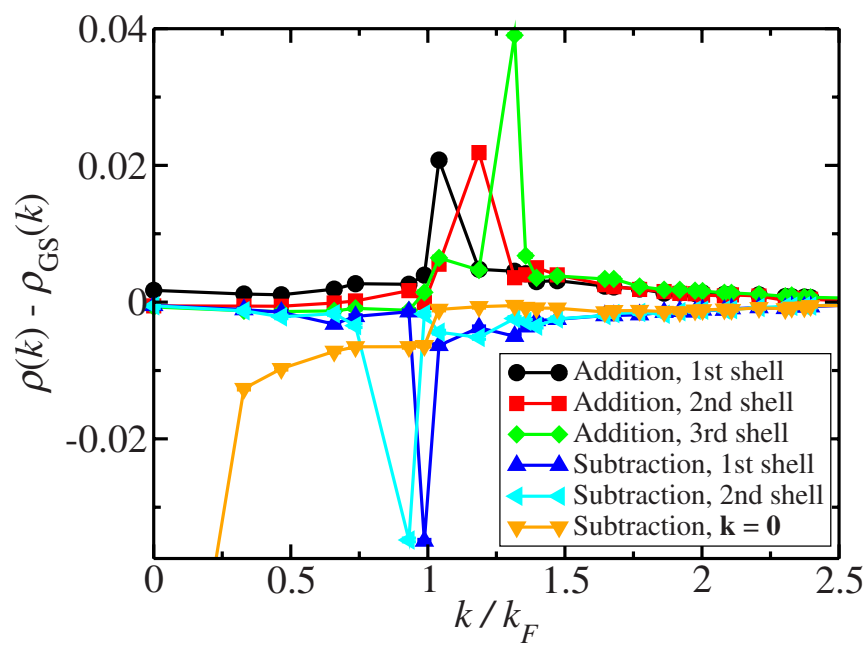

FIG. 11. (Color online) VMC momentum density $\rho(k)$ relative to the ground-state momentum density $\rho_{\mathrm{GS}}(k)$ for different excitations to a paramagnetic 58-electron HEG at $r_{s}=10$ a.u. The momentum densities are averaged over reciprocal-lattice vectors of the same length, so the height of the spike at the $|\mathbf{k}|$ at which the electron is added looks smaller when there are many reciprocallattice vectors with the same length as $|\mathbf{k}|$. 
and Physical Sciences Research Council. Computing resources were provided by the Cambridge HPCS and HPCx. We are grateful to W. M. C. Foulkes for many useful discus- sions. We thank M. Padmanabhan and Y.-W. Tan for sending us their experimental effective mass data and R. Asgari for sending us his $G W$ effective mass data.
${ }^{1}$ L. D. Landau, Sov. Phys. JETP 3, 920 (1957); 5, 101 (1957); 8, 70 (1959).

${ }^{2}$ G. F. Giuliani and G. Vignale, Quantum Theory of the Electron Liquid (Cambridge University Press, Cambridge, 2005).

${ }^{3}$ J. L. Smith and P. J. Stiles, Phys. Rev. Lett. 29, 102 (1972).

${ }^{4}$ V. M. Pudalov, M. E. Gershenson, H. Kojima, N. Butch, E. M. Dizhur, G. Brunthaler, A. Prinz, and G. Bauer, Phys. Rev. Lett. 88, 196404 (2002).

${ }^{5}$ Y.-W. Tan, J. Zhu, H. L. Stormer, L. N. Pfeiffer, K. W. Baldwin, and K. W. West, Phys. Rev. Lett. 94, 016405 (2005).

${ }^{6}$ M. Padmanabhan, T. Gokmen, N. C. Bishop, and M. Shayegan, Phys. Rev. Lett. 101, 026402 (2008); T. Gokmen, M. Padmanabhan, K. Vakili, E. Tutuc, and M. Shayegan, Phys. Rev. B 79, 195311 (2009).

${ }^{7}$ R. Asgari, B. Davoudi, M. Polini, G. F. Giuliani, M. P. Tosi, and G. Vignale, Phys. Rev. B 71, 045323 (2005).

${ }^{8}$ R. Asgari and B. Tanatar, Phys. Rev. B 74, 075301 (2006).

${ }^{9}$ Y. Kwon, D. M. Ceperley, and R. M. Martin, Phys. Rev. B 50, 1684 (1994).

${ }^{10}$ Y. Kwon, D. M. Ceperley, and R. M. Martin, Phys. Rev. B 53, 7376 (1996).

${ }^{11}$ M. Holzmann, B. Bernu, V. Olevano, R. M. Martin, and D. M. Ceperley, Phys. Rev. B 79, 041308(R) (2009).

${ }^{12}$ Y. Zhang and S. Das Sarma, Phys. Rev. Lett. 95, 256603 (2005).

${ }^{13}$ R. Asgari, T. Gokmen, B. Tanatar, M. Padmanabhan, and M. Shayegan, Phys. Rev. B 79, 235324 (2009).

${ }^{14}$ W. M. C. Foulkes, L. Mitas, R. J. Needs, and G. Rajagopal, Rev. Mod. Phys. 73, 33 (2001).

${ }^{15}$ The Fermi wave vector is $k_{F}=\sqrt{2} / r_{s}$ for the 2D paramagnetic fluid and $2 / r_{s}$ for the $2 \mathrm{D}$ ferromagnetic fluid.

${ }^{16}$ R. J. Needs, M. D. Towler, N. D. Drummond, and P. López Ríos, CASINO Version 2.1 User Manual (University of Cambridge,
Cambridge, 2008).

${ }^{17}$ J. B. Anderson, J. Chem. Phys. 65, 4121 (1976).

${ }^{18}$ N. D. Drummond, M. D. Towler, and R. J. Needs, Phys. Rev. B 70, 235119 (2004).

${ }^{19}$ P. López Ríos, A. Ma, N. D. Drummond, M. D. Towler, and R. J. Needs, Phys. Rev. E 74, 066701 (2006).

${ }^{20}$ C. J. Umrigar, K. G. Wilson, and J. W. Wilkins, Phys. Rev. Lett. 60, 1719 (1988).

${ }^{21}$ N. D. Drummond and R. J. Needs, Phys. Rev. B 72, 085124 (2005).

${ }^{22}$ C. J. Umrigar, J. Toulouse, C. Filippi, S. Sorella, and R. G. Hennig, Phys. Rev. Lett. 98, 110201 (2007).

${ }^{23}$ N. D. Drummond and R. J. Needs, Phys. Rev. B 79, 085414 (2009).

${ }^{24}$ The additional electron is inserted in orbital $\exp (i \mathbf{k} \cdot \mathbf{r})$ and allowed to correlate with the other $N$ electrons in the system. Operationally, this means that we extend the Jastrow factor and backflow function to include the extra electron, giving a wave function that is an approximation to an energy eigenstate of the $(N+1)$-electron system. We then use this wave function as the trial wave function in a DMC calculation to determine the excited-state energy within the fixed-node approximation.

${ }^{25}$ G. Rajagopal, R. J. Needs, S. Kenny, W. M. C. Foulkes, and A. James, Phys. Rev. Lett. 73, 1959 (1994).

${ }^{26}$ G. Rajagopal, R. J. Needs, A. James, S. D. Kenny, and W. M. C. Foulkes, Phys. Rev. B 51, 10591 (1995).

${ }^{27}$ The ferromagnetic data of Padmanabhan et al. (Ref. 6) were obtained in a high external magnetic field, which was not present in our calculations. However, the normal component of the magnetic field, which is the only component that affects the behavior of an ideal 2D HEG, was relatively small. 